\title{
Kenaf (Hibiscus cannabinus L.) seed oil-in-water Pickering nanoemulsions stabilised by mixture of sodium caseinate, Tween 20 and $\beta$-cyclodextrin
}

\begin{abstract}
The limit application of functional kenaf (Hibiscus cannabinus L.) seed oil in food and pharmaceutical industry owing to the poor water solubility and low storage stability can be overcome by the development of kenaf seed oil-in-water Pickering nanoemulsions. In this study, oil-in-water Pickering nanoemulsions were produced to investigate its stability by optimising emulsifier mixtures, namely sodium caseinate (SC), Tween 20 (T20) and bcyclodextrin (B-CD). The interaction effects of SC and T20 on the formation of Pickering nanoemulsions with $6-C D$ was studied and found synergistic effect among them that enhanced the stability of Pickering nanoemulsions. The optimum proportion of emulsifier mixtures obtained by employing simplex centroid mixture design was found to be $57.9 \%$ (w/w) SC, 27.6\% (w/w) T20, and 14.5\% (w/w) 6-CD, which produced Pickering nanoemulsion with mean particle size of $155.53 \mathrm{~nm}$, PDI of 0.07 and zeta-potential of $\mathbf{\imath} 46.67$ $\mathrm{mV}$. These experimental values were in accordance with the predicted value, indicating the adequacy of the fitted models. The mixture design was found to be a valuable tool to optimise and study the interaction effects of different components for the development of stable Pickering nanoemulsions.
\end{abstract}

Keyword: Kenaf (Hibiscus cannabinus L.) seed oil; Pickering nanoemulsions; High pressure homogenisation; Sodium caseinate; Tween 20; б-cyclodextrin 